\title{
Multiple epiphyseal dysplasia, Beighton type
}

INSERM

\section{Source}

INSERM. (1999). Orphanet: an online rare disease and orphan drug data base. Multiple epiphyseal dysplasia, Beighton type. ORPHA:166011

Multiple epiphyseal dysplasia, Beighton type is a skeletal dysplasia characterized by epiphyseal dysplasia (usually mild) associated with progressive myopia, retinal thinning, crenated cataracts, conductive deafness, and stubby digits. 